\title{
Zwölfte Sitzung der Soeiété belge d'ophtalmologie
}

\section{zu Brüssel am 26. April 1902}

Krá́ k e n v or s t e 11 a n ge n.

De Lantsheere-Brüssel zeigte eine@ Mascbinisten vor mit Keratoconus bei traumatischer

Neurose. Der Mascbiuist iiel während der Eisenbabnfahrt von seiner Lokomotive, hatte mehrere

Kontusionen und eine beftige Erschütterung des Kopfes; mehrere Fremdkörper in der rechten

Hornhaut. Es blieb da eine zarte Trübung zuríick mit V=2/3, während links V=1 war. Der

Kranke magerte ab, Kopf-schmerzen, Schwindelanfälle, Schlaflosigkeit, Verminderung

derlntelligenz. Sehschärfe vermindert ohne objektiven Befund bis weniger als $1 \mathrm{j} 10$ beiderseits;

etwas Blepharospasmus und Photophobie. Die Refraktion hat objektiv skiaskopisch variiert; drei Wochen nach dem Unfall etwas Myopie, nie über - 3 D.; später Hypermetropie, dann wieder Myopie, dann Hypermetropie; auch Astigmatismus. Tension immer normal. Sechzehn Monate nach dem Unfall war sine konische Corneakonstatierbar, welche sich immer starker $\Lambda$ völbte. Vortr. führt die Entstelmng des Keratoconus auf die allgemeine Schwäche zurück oder auf eine lokale Veränderung der Nerven oder Gefässe, bei dem Unfall entstanden. Die Macula corneae sitzfc an der Spitze des Keratoconus, welcher ohne Ent-zündungserscheinungen entstand. Venneman-Löwe@ sab Patient vor zwei Monaten und konstatierte den Keratoconus an beiden Augen.

Coppez-Brüssel stellte einen 19jährigen Patienten vor mit Sinusitis ethmoidalis, der ausser häufigen Nasen-Katarrhen keine speziellen Erkrankungen durchgemacht hat. Während einer Vergnügungs-reise entstand eine fötide Entleerung aus der link en Nasenhälfte, welche sich verstopfte. Am 6. April blieb er spat aus, bekam Schüttelfrost und erwachte den nächsten Tag matt, fiebernd, das linke Auge ge-schwollen, schmerzend und schlechter sehend. Zwei Tage später wurde anderswo die Highmore-Höhle eröffnet, dann der linke Sinus frontalis, ohne class die Beschwerden gehoben wurden oder dass, nach den An-gaben des Patienten, Eiter herausbefördert wurde. Am 15. April stellte Patient sich in Brüssel vor. Die Durchleuchtung gab links fur das Antrum Highmori und den Sinus frontalis eine dunklere Abstufung als rechts, obschon kein Eiter auwesend war. Das praeauriculare Ganglion war links gesclnvollen. Der Opticus war nur getrübt zu sehen; venose llyperämie des Fundus; V =1,20; Sehfeld normal. Gallet legte Jiocbmal das Antrum Highmori frei und fand nichts Abnormes; ebenso den Sinus frontalis; dann den Sinus ethmoidalis, von wo aus Eiter und ein Stuck cariöser Knochen von \pm 1 Quadratcentimeter entfernt wurde. Nor-maler Verlauf; acht Tage nachher V = nahezu normal. de Lants-heere teilt den Fall mit von ein em lõjährigen Mädchen, wo nach einer Influenza eine Entziindung des Sinus ethmoidalis entstanden war, welche

302

Sitzungsbericht. entzündlicheErscheinungen bis dreimal zurückkehrten, bis eineCurettierung des Sinus Heiluagg erzielte.

Vorträge. 
Coppez-Brüssel sprach über das BelГsche Phaenomen und zeigt Photographien vor von einem Kinde, fünf Jahre alt und mit congenitaler Diplegia facialis behaftet. Das Bell'sche Phaenomen besteht hier wie bei einem normalen Individuum: so schliesst Vortr. mit der Verwerfung von Nag el's Hypothese.

Lor-Brüssel sprach über essentielle Ophthalmomalacie. Eine 36jährige Frau litt seit acht Tagen an Schmerzhaftigkeit und Ver-minderung der Sehschärfe des línken Auges. Es bestand Thränenfluss, mässige Rötung der Bindehaut, leichtes Oedem der Lider; nach aussen von der Hornhaut war nur ein Episcleritisknoten zu bemerken; Hornhaut durchsichtig, aber beim Knoten stark nach innen eingedrückt; Epithel mit Pluorescein als normal nachgewiesen. Einträufelung von Cocain, Borsäurewaschungen. Bei der zweiten Vorstellung, zwei Wochen später, war alles normal. Vortr. nimrat den Conjunctivalknoten als Faktor an, welcher die excentrische Lage der Depression bedingte, und sieht die rapide Heilung als durch die Cocainw $1 / 8$ kung hervorgebracht an. - Stockë-Beveren- $\Lambda$ Vaas behandelte eine 42 jährige Frau wegen doppel-seitiger myopischer Netzhautablösung mit acoinisierter 10 pCt. NaCl-lösung, welche links in die Uebergangsfalte subconiunctival eiugespritzt wurde. Tags darauf eine Depression der Cornea aussen. Atropin; Bettruhe, Verband. Er nimmt als Ursache ein Austreten von Augen-flüssigkeit zur Stelle der Injektion an. Bei Átropineinträufelung verschwand die Hornhautdepression und kehrte bei einer zweiten Ein-spritzung nicht wieder.

Rutten-Lüttich sprach über ausserordentliche Ektasie des Thränensacks links bei einer 63jährigen Frau, welche seit etwa dreissig Jahren nur an dieser Seite an Dacryocystitis litt und selber die Entleerung durch Druck hervorbrachte. In den letzten zwei Jahren gelang ihr dieses nur selten: bei der Vorstellung war dieses seit zwei Monaten nicht mehr geschehen und war der Sack eigross angeschwollen. Nase und Nebenhöhlen normal. Exstirpation; Thermocauterisierung des dem Knochen anhaftendeu Teiles; Heilung mit leichtem Ectropium. Der Sack war stark verdickt, wie Leder, und mass nach der Exstirpation 37X14 Millimeter. Er enthielt einen gelblichgrünlichen íibel-riechenden Eiter.

Rutten-Lüttich sprach dann über einseitigen vorübergehenden Exophthalmus links bei einem 14jährigen Jungen. Derselbe erwachte morgens früh mit Anschwellung des linken Auges; tags darauf Au-schwellung der linken Wange, was in drei Tagen verschwand. Am siebenten Tage verschwand das Oedem der Lider, während der Exophthalmus weiterbestand; Auge nicht gerötet, frei beweglich; nichts Ab-normes in der Orbita zu bemerken, aber Propulsion des Bulbus. Pupille erweitert, aber gut reagierend: V = Finger in zwei Meter; SteUwag's und v. Graefe's Symptome anwesend. Im Fundus waren die Iläute um die Papilla nach vorn gedrängt, so dass eine falsche Excavation entstand; venose Hyperämie; durch die Kompression der linken Carotis Instrumente.

303

nichts verändert. Es wurden 2 g Jodkali pro die gegeben; ach’t Tage nachher Verschlechterung, Anschwellung der Praeauricular- und der Subinaxillarganglien; ausser dem Jodkali nun auch Quecksilbereinreibungen. Rückgang der Erscheinungen innerhalb zwei Wochen mit normaler Seh-schärfe; es ist inzwischen eine Anschwellung der Thyreoidea eingetreten; 110 Pulse. Vortr. nirnmt die Anämie und Morbus Gra〉·esi als Ursachen an, inn. so rnehr letzterer, da die Schwester des Pat. daran gestorben ist. 5. Venneman-Löwen teilt Bemerkungen über die letzten Fälle von Buphthalmus aus seiner Klinik rnit, welche sich niclit zu einem Referate eignen. Enter den Fallen befindet sich ein congenitaler; Vortr. nimmt den Namen Buphthalmus in seiner ausgedehntesten Be-deutung, so ist u. A. ein gliomatöses Auge darunter begrifí'en. Per gens. 
Instrumente.

Aus der Universitatsangenklinik Basel (Prof. Mellinger).

Ein neues aseptísehes Instrument zur Ausspülung der vorderen Kammer bei der Starextraktion.

Von

Dr. A. ERB,

Augenarzt in Lugano, gew. 1 Assistenzarzt der Augenklinik Basel.

Wir furchten in unserer Zeit der Antiseptik und Aseptik das Eingehen mit Instrumenten in die yordere Augenkammer nicht mehr so sehr wie das die älteren Ophthalmologen thaten. Vor all em müssen die Instrumente keimfrei sein.

Ausser class wir bakterielle und chemische Reize gänzlich zu ver-meiden suchen, müssen wir darauf achten, die nicht zu umgehenden mechanischen Insulte möglichst zu vermindern.

$\Lambda$ Yenn auch zurückbleibende Corticalis bei der Starextraktion jetzt nicht mehr die Gefahren mit sich bring! wie früher, so ist doch das längere Zeit gehinderte Sehen für den Patienten deprimierend und eine grosse Neigung zur Nachstarbildung vorhanden.

Aus diesen Gründen werden wir überall da, wo es sich technisch durchführen lässt, nach der Extraktion durch Entfernung der Corticalis eine möglichst schwarze Pupille zu bekommen suchen. üiejenige Methode, die dabei am schonendsten für das Auge verfährt, wird die besteu Resultate geben.

S. Seit langer Zeit existieren 2 Metboden

die Corticalismassen aus dem Auge zu entfernen; entweder ging man mit Instrumenten in die vordere Kammer ein

oder man suclite durch beliutsames Reiben mit Instrumenten oder mit dem untereu Lid auf der Hornhaut die Starreste herauszubringen.

Abgesehen von dem gefährlichen häufigen Eingehen mit Instrumenten in die Vorderkammer nehmen auch diese Metboden teilweise 